\title{
Study to Compare Progrip Mesh vs Conventional Mesh in Lichtenstein's Open Inguinal Hernia Repair
}

\author{
Mohammad Farooque Dudhwala ${ }^{1}$, Chiraag Hiran S. ${ }^{2}$, \\ Vipul Gurjar ${ }^{3}$, Honeypalsinh H. Maharaul ${ }^{4}$, Vipul Shory ${ }^{5}$ \\ ${ }^{1}$ Assiatant Professor, ${ }^{2}$ Resident, ${ }^{3}$ Professor, ${ }^{4}$ Associate Professor, ${ }^{5}$ Resident, Department of General Surgery, Dhiraj \\ General Hospital, Smt. B.K. Shah. Medical . Institute and Research Centre, Sumadeep Vidyapeeth Deemed to be \\ University (An Instituton), Waghodia, Pipariya, Vadodara, Gujarat, India
}

\begin{abstract}
Background: A hernia is protrusion of a tissue or an organ such as omentum or bowel loops through a defect in the weakened abdominal wall muscles . Amongst the various types of hernia, inguinal hernia is the most commonest. The best modality of treating an inguinal hernia is surgery and hence most common surgery done is inguinal hernia repair . Amongst the various techniques of hernia repair, Lichtenstein's tension free meshplasty is the latest, best and has become the gold standard . In 2008, COVIDIEN launched progrip mesh which is a self fixating mesh used in both inguinal and incisional hernia repair . Progrip mesh is made up isoelastic lightweight macroporous knitted monofilament polypropylene fabric that incorporates resorbablepolylactic acid fabric on one side of the mesh to provide self fixation during first few months of implantation.
\end{abstract}

Aim: The aim of this study is to compare the outcome on basis of incidence of postoperative pain and operative time of self fixating pro-grip mesh with conventional mesh in Lichtensteins open inguinal herniomeshplasty .

Objective:

- Comparing outcome in terms

- Post-operative pain

- Operative time

Conclusion: Lichtenstein's open inguinal herniomeshplasty using self-fixating Progrip mesh has an advantage over conventional mesh repair as it requires less operative time and the incidence of postoperative pain is less frequent and occurs at an even more later period compared to conventional mesh repair which at times is found to be present even at post-operative day 2 .

Keywords: Lichtensteins Inguinal herniomeshplasty, self fixating Progrip mesh.

\section{Corresponding Author:}

\section{Dr. Honeypalsinh H. Maharaul}

Associate Professor, Smt. B.K. Shah Medical Institute and Research Centre, Sumandeep Vidyapeeth Deemed to be University (An Institution), Pipariya Vadodara e-mail: honeypal.219@gmail.com

Phone: 9426345105

\section{Introduction}

An inguinal hernia occurs when any tissue,protrudes through a weak spot in the anterior abdominal wall muscles. Amongst the types of hernia, the most common type is inguinal hernia . And the best modality of treating an inguinal hernia is surgery .

Inguinal hernia can be repaired either in its old traditional modality of open hernioplasty or its new modality of laparoscopic hernioplasty . But each 
technique has got its advantage and disadvantage, despite that the main goal of any hernia repair is to provide the best and strongest repair, least recurrence rate, least possible discomfort and quickest recovery.

\section{In open method hernia can be managed by:}

- Herniotomy

- Herniorraphy

- Hernioplasty

Laparoscopic repair is the recent advancement. It can be done by 2 ways with mesh fixation and without mesh fixation and can be by two approaches :

- Total Extra Peritoneal (TEP)

- Trans Abdominal Preperitoneal (TAPP)

Inguinal hernia repair still remains a problem because of chronic groin pain following mesh repair.

One of the most commonly performed surgical procedures is inguinal hernia repair but despite being most common it is associated with long term risks of pain and discomfort. In 1996, according to a study done by Cunningham et $\mathrm{Al}$, he was the first to report $63 \%$ pain incidence after one year ${ }^{[1]}$. In a large cross sectional cohort study the rate of chronic pain was $29 \%$ at around 5 years following the surgery.

The mesh is supposed to be a foreign body implanted into our body which resulted in loss of myelinated axons, axonal, peri and endoneuronaledema and subsequently pain $^{[2]}$. The method of fixation may be an inportant causative factor for pain. For fixation most commonly used is the sutures which may cause ischaemia or nerve damage which eventually leads to pain . Mesh implantation can be done by several techniques, but the most commonly involved technique is by sutures to anchor the mesh in position and prevent its migration, curling and wrinkling. But these sutures that anchor the mesh can cause extensive tissue tension and nerve entrapment which are considered to be leading causes to prolonged post-operative pain, which is the main primary objective to be looked in our study. This problem does not solve even on application of absorbable sutures instead of non-absorbable ones.

The new progrip mesh is Isoelastic large-pore lightweighted, knitted, monofilament polypropylene fabric that incorporates microgrips which are resorbable and these only provide self-gripping fixation. It promotes the regeneration of healthy tissue inside its microgrips and shows low rate of complications with $100 \%$ self fixation ${ }^{[3][4]}$. Hence our present study is to enlighten the advantage of this self grippingprogrip mesh in reducing the incidence of chronic groin pain after inguinal hernia repair and reduce the operative time as it leads to less exposure to anaesthetic drugs.

\section{Materials and Method}

The prospective study was conducted between July 2019 to June 2020 and around 20 patients were divided into two groups with inguinal hernia and were admitted to Dhiraj General Hospital, S.B.K.S.M.I.R.C, Pipariya, Vadodara, Gujarat, India.

\section{Inclusion Criteria:}

Patients diagnosed with inguinal hernia and planned for lichtensteinsinguila hernia repair

Willing for study

Anaestheticaly fit patients .Exclusion criteria :

- Patient not willing for study.

- Paediatric age group patients

- Patient not fit anaesthetically.

Method of collection of data: This study involves 20 patients coming with cheif complaints of swelling in the inguinal region. Detailed history and clinical examination was done. Written and Informed Consent was obtained for taking part in study and for operative procedure. Patients were randomly divided into two groups $\mathrm{A}$ and $\mathrm{B}$, each group containing 10 patients each.

Group A patients were planned for Lichtenstein's repair with conventional mesh.

Group B patients planned for Lichtenstein's repair with self fixating pro-grip mesh.

Time period starting from skin incision to placing and fixation of mesh in each group will be noted.

Post-operative pain were noted according to pain score during post operative hospital stay and after discharge on 15th day and 3 months of follow up

\section{Procedure:}

Pateint in supine position following spinal anaesthesia 
Parts painted and draped: Skin incision placed approximately $1.25 \mathrm{~cm}$ above an imaginary line joining pubic tubercle and anterior superior iliac spine.

Skin and subcutaneous tissue incised and external oblique aponeurosis identified .

Small incision placed over the EOA along the fibres

External oblique aponeurosis opened upto the superior inguinal ring medially and deep inguinal ring laterally .

Inguinal canal opened and space created upto conjoint tendon medially and upturn part of inguinal ligament laterally .

Spermatic cord identified, stretched and lateralisation of cord structure done .

Defect identified of size approximately $2 \times 2 \mathrm{~cm}$ through which hernial sac was protruding .

Inferior epigastric artery identified and found to be lateral to the hernial sac (direct inguinal hernia) .

Hernial sac identified and reduced and posterior wall repair done with prolene no. 1 R.B .

Progrip mesh was taken after creating good space underneath and around the spermatic cord

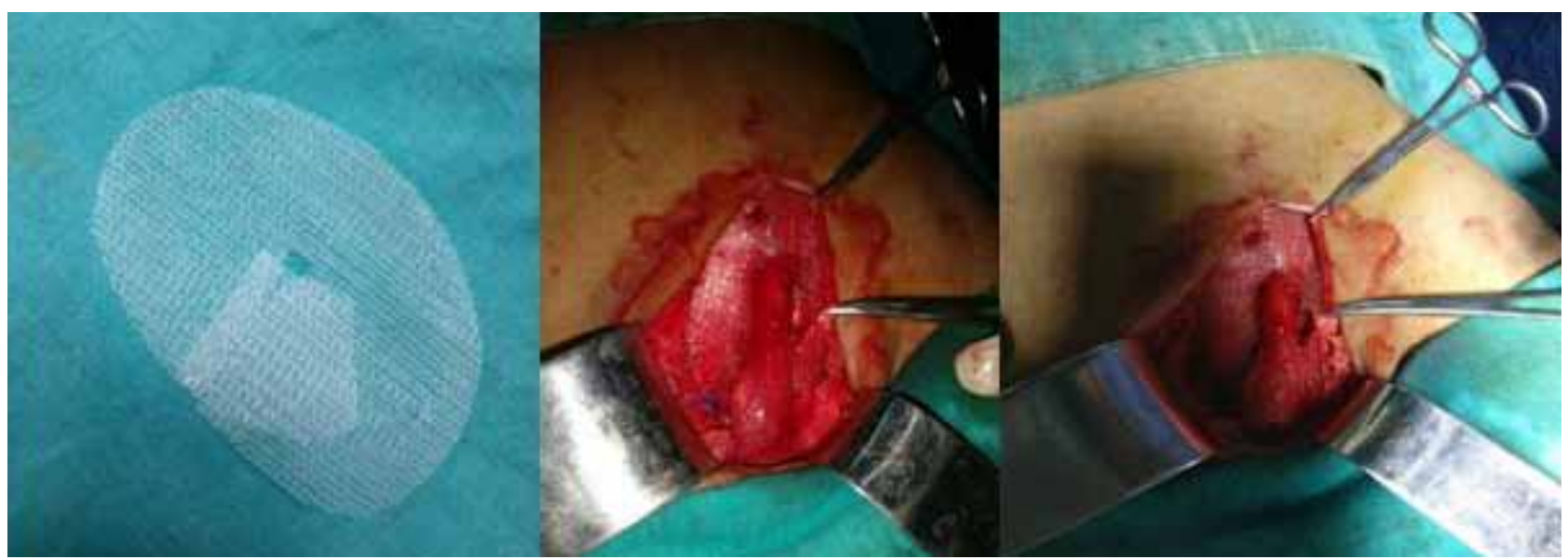

Figure 1: Showing usage of progrip mesh for hernioplasty

Master suture taken on pubic tubercle with blue mark on pro-grip mesh with prolene 1-0 R.B and the mesh spread properly.

All layers re sutured back as opened

EOA sutured in continuous fashion with prolene 2-0 R.B and artficial superficial ring created

Subcutaneous layer sutured with vicryl 2-0 R.B

Skin sutured with ethilon 2-0 R.C with subcuticular technique

Sterile dressing done after attaining hemostasis

Patient shifted to post op room as was vitally stable

\section{Discussion}

The Lichtensteins tension-free technique has gained remarkable popularity as it is easy-to-perform with less tissue dissection ${ }^{[5]}$. But questions concerning the safety of implantation of mesh for longer duration and the risk of chronic pain have always been around . Various studies have reported the rate of prolonged pain after inguinal hernia surgery to increase from $9.7 \%$ to $51.6 \%{ }^{[6]}$; and the incidence of persistent postoperative pain is reported to be as high as $6 \%$ which hinders with daily activities of the patient.

The reason for such pain is unclear . But it can be due to:

- Mesh material that is its structure and interaction with tissue 
- Scarring

- At times nerve damage

- Entrapment by sutures

Because of these facts, fixation of the mesh with sutures during surgery continues to be an important factor to be thought and discussed about.

Hence a new self-gripping mesh (Progrip) has been developed in 2008 by COVIDIEN .

This self-gripping mesh is made of: Isoelastic large-pore lightweighted, knitted, monofilament polypropylene fabric that incorporates microgrips which are resorbable and these only provide self-gripping fixation in the initial few months of implantation ${ }^{[7]}$.

The microgripsare: 1-mm projections that are club in shape and are made up of polylactic acid which is biodegradable.
The microgrips integrate for upto $0.5 \mathrm{~mm}$ into the tissue and provides stronger tissue incorporation at almost around 5 days.

Hence because of these features available in the self fixatingprogrip mesh can be placed in position safely and proper without the requirement for sutures which can penetrate underlying tissues and might damage cutaneous nerves $^{[8]}$.

\section{Result}

Operative time : Conventional mesh repair on an average takes around 35.5 minutes starting from skin incision to closure, whereas progrip mesh repair takes only around $20 \mathrm{mins}$.

Postoperative pain : as noted in these patients, after conventional mesh repair the GROUP B complaints of chronic pain even after 3 months. Whereas in GROUP A progrip mesh repaired patients they were pain free after post operative day 2 and on follow up after 3 months.

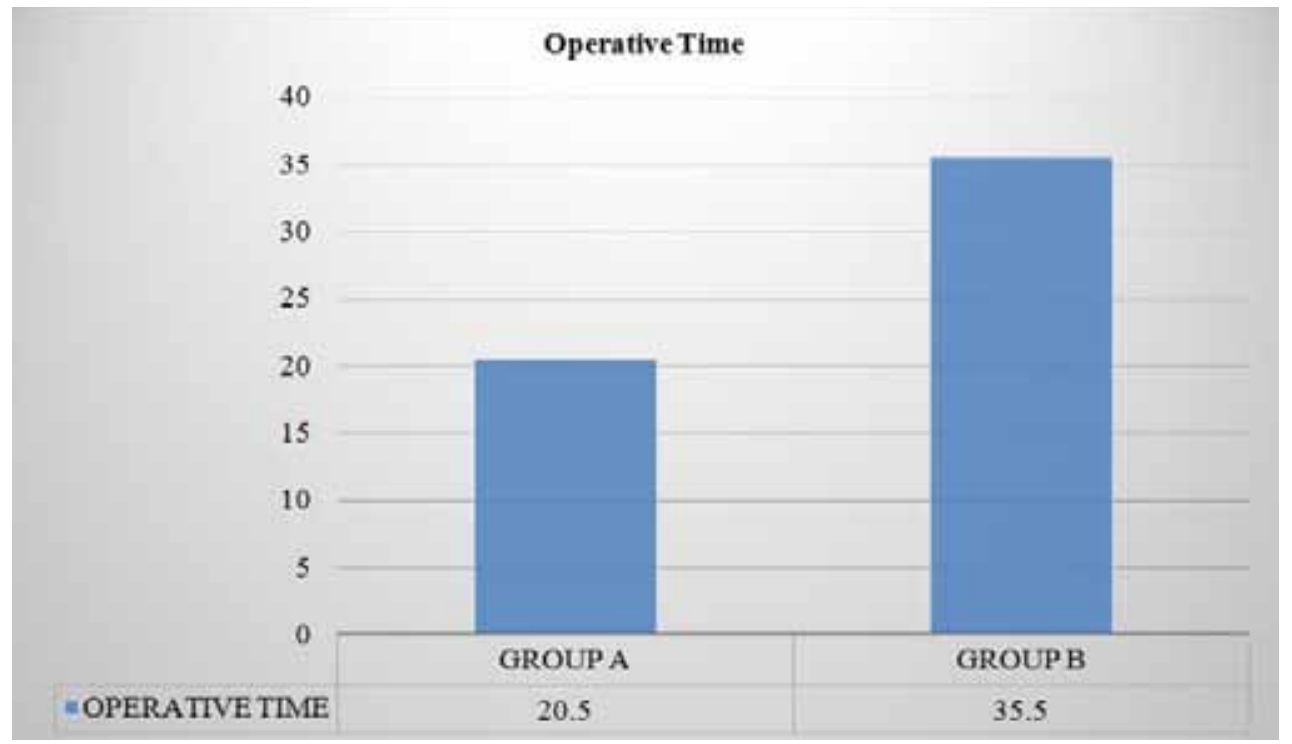

Figure 2: Showing time variation between the operative time between conventional hernioplasty and usage of progrip mesh.

\section{Conclusion}

Lichtenstein's open inguinal herniomeshplasty using self-fixating Progrip mesh has an advantage over conventional mesh repair as it requires less operative time and the incidence of post-operative pain is less frequent, mostly painless and occurs at an even more later period compared to conventional mesh repair which at times is found to be present even at post-operativd day 2 .
Ethical Clearance: Taken from sumandeep vidyapeeth institutional ethics committee

Source of Funding: Self

Conflict of Interest: Nil.

\section{References}

1. J Cunningham, W J Temple, P Mitchell, J A Nixon, 
R M Preshaw, N A Hagen et al . Cooperative Hernia Study. Pain in the Postrepair Patient . Ann surgery, 1996 Nov; 224(5):598602.

2. Lichtenstein ME. The custom-tailored inguinal hernia repair. J Okla State Med Assoc 1954; 47:222-4.

3. Dynamic implant for inguinal hernia repair in porcine experimental model. A feasibility study. Hernia 2009; 13:S1-332.

4. Dynamic autostatic implant for inguinal hernia repair. Early results in an explorative cohort of patients. Hernia 2010; 14:S55-100.

5. Nienhuijs S, Staal E, Strobbe L, Rosman C, Groenewoud H, Bleichrodt R. Chronic pain after mesh repair of inguinal hernia: a systemic review. Am J Surg 2007; 194:394-400.

6. Merskey H, Bogduk N. Classification of chronic pain: descriptions of chronic pain syndromes and definitions of pain terms. In, Task force on Taxonomy. IASP, 2nd edition. Seattle, WA: IASP press, 1994; 209-14.

7. Awad SS, Fagan SP. Current approaches to inguinal hernia repair. Am J Surg 2004; 188 (6A Suppl):9S16S.

8. Singh S. Innovative and regenerative hernia repair with dynamic implants. IHS Newsletter 2012; 6(2): 5- 8 . 